\title{
Subjective Technology Adaptivity Predicts Technology Use in Old Age
}

\author{
Stefan T. Kamin Frieder R. Lang Anja Beyer \\ Institute of Psychogerontology, Friedrich-Alexander University Erlangen-Nürnberg (FAU), Nuremberg, Germany
}

\section{Keywords}

Older adults · Gerontechnology · Technology use .

Motivation - Subjective Technology Adaptivity Inventory .

Aging and technology

\begin{abstract}
Background: To date, not much is known about the psychological and motivational factors underlying technology use in late life. What are the interindividual determinants that lead older adults to invest in using technological innovations despite the age-related physiological changes that impose challenges on behavioral plasticity in everyday life? Objective: This research explores interindividual differences in subjective technology adaptivity - a general technology-related motivational resource that accounts for technology use in late life. More specifically, we investigate the influence of this factor relative to demographic characteristics, personality traits, and functional limitations in a longitudinal sample of community-dwelling older adults. Methods: We report results from a paper-and-pencil survey with 136 older adults between 59 and 92 years of age (mean $=71.4, \mathrm{SD}=$ 7.4). Of those participants, 77 participated in a 2-year followup. We assessed self-reports of technology use, subjective technology adaptivity, functional limitations, and the per-
\end{abstract}

\section{KARGER}

(C) 2017 S. Karger AG, Basel

E-Mail karger@karger.com

www.karger.com/ger sonality traits openness to new experiences and neuroticism. Results: Higher levels of subjective technology adaptivity were associated with technology use at the first measurement as well as increased use over the course of 2 years. Conclusions: Subjective technology adaptivity is a significant predictor of technology use in old age. Our findings contribute to improving the understanding of interindividual differences when using technological innovation in late life. Moreover, our findings have implications in the context of user involvement and may contribute to the successful development of innovative technology for older adults.

(c) 2017 S. Karger AG, Basel

\section{Introduction}

Technology use in old age is often explored with respect to potentials for improving well-being and quality of life [1]. However, keeping up with new technological products in daily life can be challenging for older adults who are often late adopters of technological innovation [2, 3]. This is due to the required adaption to technological demands (e.g., using a software interface, changing hearing aid settings). Such an adaptation necessitates an investment of sensory, cognitive, and motor resources $[4,5]$, 
which are often challenged with increasing age. Although there is a good understanding of the physiological and cognitive factors that are associated with interindividual differences in technology use in late life [6-8], not much is known about the role of motivational resources influencing the change and plasticity of usage behavior among older adults. In this research, we explore the self-reported change in technology use over the course of 2 years and its association with subjective technology adaptivity, a motivational resource related to technology use in old age.

Our approach to subjective technology adaptivity aims to explain why some older adults are more likely to make use of their technological environments than others. Previous research in this domain has investigated specific motives toward the use of technology, for example with respect to emotionally relevant benefits or communication goals $[9,10]$. Also, technology acceptance models have been widely used to explore behavioral intentions related to specific devices $[11,12]$. The approach used in the present study represents a broader perspective of adaptive functioning in technological environments. It builds on assumptions derived from the ecological model of aging [13] suggesting that persons with higher personal competence are better able to benefit from environmental resources and opportunities in everyday life than persons with lower competence. Consequently, we defined subjective technology adaptivity as a motivational resource that involves a sense of personal competence in response to perceived potentials and demands of technological environments in late life [14]. We argue that interindividual differences in subjective technology adaptivity are associated with 3 motivational dimensions: (1) Perceived adaptive utility involves focusing on benefits when using technology. This dimension posits that older adults are motivated to use technological products and solutions if they perceive them as beneficial for producing changes in their environment. From a motivational perspective of adaptive lifespan development [15], such benefits are related to environmental mastery, autonomy, and behavioral control in everyday life. Empirical evidence shows that positive or negative evaluations of specific devices depend on the perception of benefits (i.e., the ability to pursue control over the social environment) [9]. Moreover, it is known that perceptions of usefulness are associated with the acceptance and usage behavior of specific products [11]. (2) Technology-related goal engagement pertains to the investment of behavioral and cognitive resources when using technology [5]. This dimension refers to a person's capacity to facilitate the attainment of goals [15]. For example, using a new device often requires a maintained or intensified effort, particularly in the face of challenges, such as usability problems. Accordingly, we argue that processes of goal engagement are crucial for the transformation of intentions of technology use into actual usage behavior. (3) Perceived safety of technology relates to a sense of safety and trustworthiness while using technology. New technological developments often raise privacy and security concerns among older adults [16, 17], which may reflect feelings of not being in control of oneself. In the same vein, it is known that worries and anxieties play a critical role for using technology in late life $[18,19]$. Consequently, we submit that a stronger sense of safety reflects a protective resource against potential risks that are associated with technological environments.

The described domains are interrelated and conjointly serve to maximize adaptive functioning in technological environments. Their covariation is caused by the higherorder factor of subjective technology adaptivity, which has been defined as a general technology-related motivational resource that accounts for positive outcomes when interacting with technological environments in late life (e.g., usage behavior). This assumption has been supported in previous research, showing that higher levels of subjective technology adaptivity were associated with higher levels of perceived competence with technology and greater use of technology among older adults [14].

Such findings, however, are based on cross-sectional data and, therefore, it remains an open question whether this construct may also predict changes in technology use. Thus, the focus of our research was to examine whether subjective technology adaptivity predicts changes in the use of technology among older adults over the course of 2 years. In accordance with previous findings and the ecological model of aging $[13,14]$, we expected that persons with higher levels of subjective technology adaptivity would report more technology use at the first measurement occasion and increased use 2 years later at the follow-up assessment. We hypothesized that these associations would be robust above and beyond the effects of other possible predictor variables. Consequently, we explored the influence of demographic characteristics, functional limitations, and personality traits. Research has indicated that the use of technology is negatively associated with higher age and being female, and positively associated with higher education $[6,18]$. Moreover, it is known that functional limitations are associated with lower technology use among older adults [7]. For example, pressing a button requires precise movement control and accuracy; having vision impairments makes it diffi-
386

Gerontology 2017;63:385-392 DOI: $10.1159 / 000471802$
Kamin/Lang/Beyer 
cult to read text on displays [4]. With respect to the relationship between technology and personality, findings are typically mixed. Examples include studies that have found positive associations between technology use and openness [20,21], while others have not [22]. One issue might be that research in this domain often focuses on different products, applications, and usage scenarios (e.g., social networking or e-commerce), which makes it difficult to compare findings across studies. Also, most research has been done with younger adults. The question of how personality accounts for usage behavior in old age is still left open. In the current study, we focused on openness and neuroticism, both reflecting relatively stable dispositions of a person's personality that account for consistent behavioral patterns in daily life [23]. We hypothesized, when showing higher levels of openness, that older adults are more likely to seize new contextual opportunities, which, in turn, may also relate to the use and implementation of technological innovation in everyday life. On the other hand, higher levels of neuroticism may be associated with concerns about potential risks and dangers of modern technology, which may result in lower utilization of technology among older adults.

\section{Methods}

\section{Participants and Procedure}

Analyses were performed on data from a survey study that were part of the German EMN-Moves project. The project aimed at developing mobility-supporting technology for community-dwelling older adults living in the Nuremberg metropolitan region, $\mathrm{Ba}$ varia. The included participants were at least 59 years old at recruitment in 2012 and were asked to complete paper-and-pencil questionnaires over the course of the project. We used data from 2012 (time 1, T1) and a 2-year follow-up in 2014 (time 2, T2). The initial sample included 136 participants - 75 women (55\%) and 61 men $(45 \%)$ - with an average age of 71.4 years $(S D=7.4)$. Of these participants, 77 (57\%) participated in the follow-up assessment, 50 (37\%) refused participation, and $9(6 \%)$ could not be contacted or provided insufficient data. The sample characteristics are reported in Table 1.

\section{Measures}

In this study, we used paper-and-pencil questionnaires with self-report measures of technology use, subjective technology adaptivity, personality, functional limitations, and demographic characteristics. The descriptive statistics are reported in Table 1.

Subjective technology adaptivity was assessed using the Subjective Technology Adaptivity Inventory [14]. This inventory includes 12 items that are related to 3 different domains ( 4 items per domain) to which participants indicated their agreement on a 5 -point scale (from $1=$ do not agree to $5=$ absolutely agree). Examples of items are: "Using modern technology helps me to be more efficient in my daily routines" (perceived adaptive utility), "I
Table 1. Descriptive statistics of the study sample $(n=136)$

\begin{tabular}{lcc}
\hline Variables & $\begin{array}{l}\text { Mean (SD) } \\
\text { or \% }\end{array}$ & Range \\
\hline Age, years & $71.4(7.4)$ & $59-92$ \\
Male gender & $45 \%$ & \\
Higher education & $1.7(1.1)$ & $0-4$ \\
Functional limitations & $2.9(2.5)$ & $0-10$ \\
Openness & $3.3(1.0)$ & $1-5$ \\
Neuroticism & $2.9(0.9)$ & $1-5$ \\
Subjective technology adaptivity & $2.9(1.1)$ & $1-5$ \\
Technology use at T1 & $2.0(1.5)$ & $0-6$ \\
Technology use at T2 & $2.1(1.6)$ & $0-6$ \\
Change in technology use from T1 to T2 & & \\
$\quad$ Technology use decreased & $26 \%$ & \\
$\quad$ Technology use remained & $55 \%$ & \\
Technology use increased & $19 \%$ & \\
\hline
\end{tabular}

T1, time $1 ;$ T2, time $2 .{ }^{\mathrm{a}} n=77$.

put in more effort when a new device is more difficult to use than expected" (technology-related goal engagement), and "I believe that new technology conforms to safety standards" (perceived safety of technology). We calculated the mean of all items to indicate the higher-order construct of subjective technology adaptivity. Cronbach a for this scale was 0.95 .

Technology use was assessed at both measurement points with questions about the actual use of 8 different technological devices related to information and communication (e.g., smartphone, Bluray player, e-book reader, media player). Participants indicated whether or not they used a specific device. We generated scores at baseline and at the follow-up assessment by summing up the number of used devices.

Change in technology use was calculated by subtracting technology use at $\mathrm{T} 1$ from use at $\mathrm{T} 2$. The resulting score was recoded into 3 dummy variables that indicated whether technology use decreased, remained the same, or increased over 2 years. Finally, we used these variables to generate an ordered 3-level variable of change in technology use ( $0=$ use decreased; $1=$ use remained; $2=$ use increased).

Functional limitations were assessed with 13 items from the Housing Enabler instrument [24]. Participants indicated the presence or absence of different functional limitations (e.g., visual impairment, hearing loss, difficulty in moving the head). A sum score was generated as an indication of the total magnitude of functional limitations.

Openness and neuroticism were assessed with a short version of the Big Five Inventory [25]. Each domain included 3 items to which participants indicated their agreement on a 5-point scale (from $1=$ do not agree to $5=$ absolutely agree). Mean-weighted scores of the items were generated for each domain. Cronbach $a$ was 0.67 for openness and 0.54 for neuroticism.

Sociodemographic variables included age in years (T1), gen$\operatorname{der}(0=$ female; $1=$ male $)$, and educational status $(0=$ no school degree; 1 = secondary school degree; 2 = intermediate school degree; 3 = upper secondary school degree; 4 = academic degree). 
Table 2. Logistic regression predicting attrition at T2 $(n=136)$

\begin{tabular}{lccc}
\hline Predictors & $B$ & SE & OR \\
\hline Age & -0.06 & 0.03 & 0.95 \\
Subjective technology adaptivity & -0.14 & 0.22 & 0.87 \\
Technology use at T1 & -0.20 & 0.18 & 0.82 \\
Male gender & $1.54^{\mathrm{b}}$ & 0.46 & 4.66 \\
Higher education & $-0.66^{\mathrm{b}}$ & 0.23 & 0.52 \\
Functional limitations & $0.32^{\mathrm{b}}$ & 0.10 & 1.38 \\
Openness & -0.40 & 0.22 & 0.67 \\
Neuroticism & -0.26 & 0.24 & 0.77 \\
\hline
\end{tabular}

$B$, logistic regression coefficient; SE, standard error; OR, odds ratio; $\mathrm{T} 1$, time $1 ; \mathrm{T} 2$, time $2 .{ }^{\mathrm{b}} p<0.01$.

\section{Analytical Strategy}

The analytical strategy was conducted in 3 steps. First, we compared means and frequency distributions of all variables between 2 groups of participants: people in the first group completed the follow-up, and the ones in the second group were lost to follow-up. We also estimated a logistic regression model to predict the probability of attrition. This strategy allowed us to explore whether missing data in our study were completely random or related to sample characteristics [26]. Second, we computed bivariate correlations and multiple regressions to explore the associations between the variables in our study. Two regression models with robust standard errors were estimated to examine the relationship between subjective technology adaptivity and use of technology at $\mathrm{T} 1$ and T2 while controlling for sociodemographic variables, functional limitations, and personality traits. Since we were interested in the change in technology use, we also controlled the longitudinal model for the initial use of technology. Finally, we estimated an ordered logistic regression model with robust standard errors. This model had the same form as the first regression model, but included the ordered 3-level variable of change in technology use as an outcome. We used this approach to directly predict the odds of reporting a greater number of devices used between T1 and T2. All analyses were conducted with Stata14 [27].

\section{Results}

\section{Attrition Analysis}

Participants who did not participate in the follow-up reported lower technology use at T1 (nonparticipants: mean $=1.63, \mathrm{SD}=1.32$; participants: mean $=2.21, \mathrm{SD}=$ 1.58 ; $p<0.05$ ), lower openness (nonparticipants: mean $=$ 3.03, $\mathrm{SD}=1.13$; participants: mean $=3.53, \mathrm{SD}=0.90$; $p<0.01$ ), more functional limitations (nonparticipants: mean $=3.56, \mathrm{SD}=2.60$; participants: mean $=2.34, \mathrm{SD}=$ 2.20; $p<0.01$ ), and less education (nonparticipants: mean $=1.32, \mathrm{SD}=0.88$; participants: mean $=1.92, \mathrm{SD}=$ $1.16 ; p<0.01)$ than those who participated in the follow- up assessment. Additionally, most of the nonparticipants were males (nonparticipants: 58\%; participants: $35 \%$; $\left.\chi^{2}(1)=6.87, p<0.01\right)$.

Table 2 shows the logistic regression coefficients and odds ratios for each variable predicting the probability of attrition at T2. The findings indicate that being male and having more functional limitations were associated with a higher probability of attrition. In contrast, older adults with higher education were more likely to participate in the follow-up assessment. The results point to the need to adjust for possible attrition bias when interpreting longitudinal data.

\section{Correlation Analysis}

Bivariate correlations in Table 3 indicate that the use of technology at T1 was negatively associated with chronological age and positively associated with being male, higher education, openness, and subjective technology adaptivity. Technology use at T2 was negatively associated with chronological age and positively associated with higher education and subjective technology adaptivity. We did not observe any significant correlations between subjective technology adaptivity and other personal or demographic variables (including age), except for openness to new experiences. Regarding the change measures, a decrease in technology use was negatively associated with chronological age; reporting the same number of devices was associated with being older and having higher levels of neuroticism; and an increase in the number of devices used was positively correlated with being male and subjective technology adaptivity, and negatively correlated with neuroticism. Finally, we observed a high stability in the number of reported devices between both measurement occasions.

\section{Linear Regression Models}

Table 4 shows findings from 2 regression models. The first model explored whether technology use at T1 could be predicted by subjective technology adaptivity, age, gender, education, functional limitations, openness, and neuroticism. The findings indicate that the number of used devices decreased for each year of age and increased for each 1-unit increase in educational level and subjective technology adaptivity. The observed correlation patterns between technology use, being male, and openness were no longer significant when controlling for all predictors in the regression. We conducted a supplementary analysis to determine whether other variables would become significant when excluding subjective technology adaptivity from the model. It was shown that openness
388

Gerontology 2017;63:385-392

DOI: $10.1159 / 000471802$
Kamin/Lang/Beyer 
Table 3. Correlations between all variables at $\mathrm{T} 1(n=136)$ and $\mathrm{T} 2(n=77)$

\begin{tabular}{|c|c|c|c|c|c|c|c|c|c|c|c|c|}
\hline & & 1 & 2 & 3 & 4 & 5 & 6 & 7 & 8 & 9 & 10 & 11 \\
\hline 1 & Age & - & & & & & & & & & & \\
\hline 2 & Male gender & -0.05 & - & & & & & & & & & \\
\hline 3 & Higher education & $-0.27^{\mathrm{b}}$ & 0.08 & - & & & & & & & & \\
\hline 4 & Functional limitations & $0.19^{\mathrm{a}}$ & -0.14 & -0.10 & - & & & & & & & \\
\hline 5 & Openness & -0.11 & -0.02 & 0.15 & -0.05 & - & & & & & & \\
\hline 6 & Neuroticism & -0.04 & $-0.20^{\mathrm{a}}$ & -0.11 & $0.27^{\mathrm{b}}$ & -0.11 & - & & & & & \\
\hline 7 & Subjective technology adaptivity & -0.15 & 0.16 & 0.13 & -0.01 & $0.40^{\mathrm{c}}$ & -0.13 & - & & & & \\
\hline 8 & Technology use at T1 & $-0.35^{\mathrm{c}}$ & $0.19^{\mathrm{a}}$ & $0.35^{\mathrm{c}}$ & -0.16 & $0.28^{c}$ & -0.10 & $0.44^{\mathrm{c}}$ & - & & & \\
\hline 9 & Technology use at $\mathrm{T} 2$ & $-0.32^{\mathrm{b}}$ & 0.19 & $0.39^{c}$ & -0.14 & 0.04 & -0.19 & $0.41^{\mathrm{c}}$ & $0.83^{\mathrm{c}}$ & - & & \\
\hline 10 & Technology use decreased & $-0.23^{\mathrm{a}}$ & -0.00 & 0.07 & -0.10 & 0.14 & -0.05 & -0.06 & $0.45^{\mathrm{c}}$ & 0.01 & - & \\
\hline 11 & Technology use remained & $0.26^{\mathrm{a}}$ & -0.20 & -0.13 & 0.22 & -0.15 & $0.22^{\mathrm{a}}$ & -0.15 & $-0.49^{c}$ & $-0.43^{c}$ & $-0.65^{\mathrm{c}}$ & - \\
\hline 12 & Technology use increased & -0.07 & $0.26^{\mathrm{a}}$ & 0.09 & -0.16 & 0.04 & $-0.22^{\mathrm{a}}$ & $0.26^{\mathrm{a}}$ & 0.12 & $0.53^{c}$ & $-0.29^{\mathrm{a}}$ & $-0.54^{\mathrm{c}}$ \\
\hline
\end{tabular}

T1, time 1 ; T2, time $2 .{ }^{\mathrm{a}} p<0.05,{ }^{\mathrm{b}} p<0.01,{ }^{\mathrm{c}} p<0.001$.

Table 4. Multiple regression models predicting technology use at T1 $(n=136)$ and T2 $(n=77)$

\begin{tabular}{|c|c|c|c|c|c|c|}
\hline \multirow[t]{2}{*}{ Predictors } & \multicolumn{3}{|c|}{ Technology use at T1 } & \multicolumn{3}{|c|}{ Technology use at T2 } \\
\hline & $B$ & SE & $\beta$ & $B$ & SE & $\beta$ \\
\hline Subjective technology adaptivity & $0.43^{\mathrm{c}}$ & 0.11 & 0.32 & $0.25^{\mathrm{a}}$ & 0.10 & 0.16 \\
\hline Age & $-0.04^{\mathrm{b}}$ & 0.01 & -0.21 & 0.00 & 0.02 & 0.02 \\
\hline Male gender & 0.29 & 0.22 & 0.10 & 0.33 & 0.25 & 0.10 \\
\hline Higher education & $0.31^{\mathrm{b}}$ & 0.10 & 0.23 & 0.14 & 0.11 & 0.10 \\
\hline Functional limitations & -0.05 & 0.04 & -0.08 & 0.03 & 0.04 & 0.04 \\
\hline Openness & 0.13 & 0.11 & 0.09 & -0.17 & 0.13 & -0.09 \\
\hline Neuroticism & 0.02 & 0.13 & 0.01 & -0.12 & 0.10 & -0.07 \\
\hline Technology use at T1 & & & & $0.76^{\mathrm{c}}$ & 0.09 & 0.74 \\
\hline Adjusted $R^{2}$ & & & 0.31 & & & 0.70 \\
\hline
\end{tabular}

$B$, unstandardized regression coefficient; SE, robust standard error; $\beta$, standardized beta coefficient. ${ }^{\mathrm{a}} p<0.05,{ }^{\mathrm{b}} p<0.01,{ }^{\mathrm{c}} p<0.001$.

became a significant predictor of technology use $(B=$ $0.31, t=0.289, p<0.01)$, while the effects of age $(B=-0.05$, $t=-3.47, p<0.001)$ and education $(B=0.33, t=2.84, p<$ $0.01)$ remained at the same level. The second model predicted technology use at T2 and included initial use as an additional covariate. As can be seen, subjective technology adaptivity was, once again, a significant predictor of technology use. Moreover, baseline technology use turned out to be the most important predictor in the model. Again, we repeated the analysis and excluded subjective technology adaptivity from the model. All regression coefficients and significance levels remained mostly the same, except for a slightly stronger effect of prior use $(B=0.82, t=8.80, p<0.001)$. Finally, we repeated the second regression model with the inclusion of all variables using multiple imputations of missing data for use of technology at T2 [28]. This approach was used to ensure that sample attrition was not affecting the findings. The unstandardized regression coefficient for subjective technology adaptivity remained robust $(B=0.28, t=2.71, p<$ 0.05).

\section{Ordered Logistic Regression}

Table 5 shows results from an ordered logistic regression model which was estimated to predict the probability of change in use of technology across the course of 2 years. This model indicates that for each 1 -unit increase in subjective technology adaptivity, the odds of reporting an increased use versus a decreased or an identical use were 1.64 times greater. 
Table 5. Ordered logistic regression predicting change in technology use $(n=77)$

\begin{tabular}{lccc}
\hline Predictors & $B$ & SE & OR \\
\hline Subjective technology adaptivity & $0.49^{\mathrm{a}}$ & 0.22 & 1.64 \\
Age & 0.07 & 0.04 & 1.07 \\
Male gender & 0.48 & 0.56 & 1.59 \\
Higher education & 0.14 & 0.25 & 1.15 \\
Functional limitations & -0.01 & 0.09 & 0.99 \\
Openness & -0.29 & 0.28 & 0.75 \\
Neuroticism & -0.11 & 0.24 & 0.90 \\
\hline
\end{tabular}

$B$, ordered logistic regression coefficient; SE, robust standard error; OR, odds ratio. ${ }^{\mathrm{a}} p<0.05$.

\section{Discussion}

We examined the association between subjective technology adaptivity and change in technology use in a longitudinal sample of older adults over 2 years. Older adults who reported higher subjective technology adaptivity were more likely to utilize technology at the first measurement. Moreover, the findings indicate that subjective technology adaptivity predicted linear change in the number of reported devices used as well as the odds of reporting increased use from $\mathrm{T} 1$ to $\mathrm{T} 2$. These findings have theoretical implications and are relevant for the development of technological products for older adults.

Technology use in old age is a complex phenomenon [18]. It depends on a multitude of factors that reflect the heterogeneity of aging processes both between and within persons. Research in the field has accumulated a rich body of evidence showing that physiological and cognitive domains of functioning play a critical role for the interaction with technological environments in old age [68]. Our findings add to this growing literature in 3 ways. First, our research suggests that the ability to make use of technological innovation in late life does not only depend on the variability of declines in physiological and cognitive domains, but also on interindividual differences in motivational resources related to subjective technology adaptivity. Second, our approach to subjective technology adaptivity is based on ecological and motivational perspectives on aging $[13,15]$. Thus, this research further contributes to the integration of gerontological theories in the understanding of technology use in old age [1]. More specifically, it points to the behavioral plasticity of older adults when dealing with environmental challenges and demands [29]. Similarly, our approach relates to eco- logical concepts, such as proactivity and agency, which help us to understand the conditions under which older adults make use of their environments and the opportunities provided to them $[13,30,31]$. Finally, subjective technology adaptivity is different from the concept of technology acceptance that has become increasingly popular in research on aging and technology $[2,11]$. Technology acceptance models have emerged from the field of information systems research and aim to explain behavioral intentions toward the use of specific devices. The approach to subjective technology adaptivity goes beyond the evaluation of specific device characteristics (e.g., the ease of use). It takes a broader perspective and explains why some older adults may be able to benefit more from the opportunities offered by technological environments than others. Consequently, both approaches may cross-fertilize each other, for example through exploration of the source causing interindividual intentional differences in specific usage scenarios (e.g., Ambient Assisted Living or smart home technology).

Although this research is focused on the role of subjective technology adaptivity, the findings also shed light on the relationship between personality traits and the use of technology in old age. For example, higher levels of neuroticism were positively correlated with identical technology use and negatively correlated with increased utilization of technology between T1 and T2. Moreover, there was a positive correlation between the number of reported devices at $\mathrm{T} 1$ and openness to new experiences, but these effects were not significant in the regression models and disappeared when controlling for subjective technology adaptivity and prior use. It is noteworthy, however, that there was a strong correlation between openness and subjective technology adaptivity. Also, openness was a significant predictor of technology use at $\mathrm{T} 1$ when subjective technology adaptivity was excluded from the model. This may point to a possible mediation hypothesis: openness predicts subjective technology adaptivity, which in turn predicts use of technology. From the perspective of personality research [23], subjective technology adaptivity might act as a proxy variable between broad differences in a person's personality and specific behavior. Thus, technology-related motivational resources may be more closely related to actual technology use than are traits. Consequently, interindividual differences in traits may be crucial for a better understanding of why some older adults report higher levels of subjective technology adaptivity than others. Future research is needed to explore the nature of these constructs and the associations between them.
Kamin/Lang/Beyer 
With respect to practical implications, our findings may encourage engineers and designers to consider the role of motivational and psychological resources in the development of technological products. To date, a rich body of knowledge shows how to consider age-related loss in sensory, cognitive, and motor functioning and to adapt technology development accordingly [4]. While this approach has contributed to create usable products that meet the specific needs of older adults, little is known about how interindividual differences in psychological and motivational resources may influence preferences and requirements of different user groups. For example, a previous study in the context of user involvement found that higher levels of subjective technology adaptivity predicted positive evaluations of different products among older adults [32]. Such findings indicate the potential usefulness of this measure for the differentiation of target groups in the user-centered design process. Future research could explore in what ways differences in subjective technology adaptivity may define the direction that technology development should take (e.g., range of functions, design or level of autonomy).

When generalizing the findings of this research there a few caveats that ought to be considered. First, this study is based on a small sample of community-dwelling older adults of 3 cities in Northern Bavaria, Germany. It is possible that findings would be different for other groups of older adults, depending on language, culture, or regional disparities. For example, research based on nationally representative data from the US has shown that older adults with limited physical capacity are less likely to use modern technology [7]. We found no association between functional limitations and technology use in our study, which might be an indicator of sample selectivity. Second, the current research relied on self-report measures that may have introduced response bias. Also, our measure of technology use reflects only an approximation of actual usage behavior. It does not capture information about usage frequencies or demands that are associated with specific devices. Further research is needed to replicate our findings with experimental approaches containing objective measures of usage behavior, such as task performance. Third, although subjective technology adaptivity and previous use were the only predictors of change in technology use over time, there was still a significant effect of age at the first measurement. Thus, the variables in this study did not fully account for age-related differences, and this underlines the complexity of technology use in late life [18]. Clearly, there is a multitude of socioeconomic, biographical, and cognitive factors that go beyond the scope of this research. Future research

Subjective Technology Adaptivity Predicts Technology Use should explore whether subjective technology adaptivity is a robust predictor of technology use when accounting for objective measures of physiological and cognitive functioning as well as socioeconomic factors.

Finally, a cautious note is warranted concerning the directionality of associations between the variables in this study. Although we used a longitudinal approach, it is not entirely clear whether higher levels of subjective technology adaptivity lead to greater technology use or vice versa. For example, a stronger involvement with technology may also affect the perceived personal competence when confronted with technological demands. In our independent analyses, we found no evidence for such an effect. However, sample size is an issue when investigating longitudinally dynamic and reciprocal interactions. Future research may explore how changes in subjective technology adaptivity and involvement with technology are jointly related to each other.

Despite these limitations, the present study contributes to the literature by showing that the combined influence of benefit perceptions, investment of resources, and safety beliefs is associated with greater use of technology among older adults. We hope that such findings contribute to an improved understanding of interindividual differences that lead older adults to keep up with new technological solutions and products in everyday life.

\section{Acknowledgments}

This research was supported by the German Federal Ministry of Education and Research (16SV5703). We thank Pierre-Marc Paré and Franziska Damm for valuable comments on the manuscript.

\section{Disclosure Statement}

The authors state that there are no conflicts of interest.

$\begin{aligned} \text { References } \quad & \text { Schulz R, Wahl HW, Matthews JT, De Vito } \\ & \text { Dabbs A, Beach SR, Czaja SJ: Advancing the } \\ & \text { aging and technology agenda in gerontology. } \\ & \text { Gerontologist 2015;55:724-734. } \\ 2 & \text { Charness N, Boot WR: Technology, gaming, } \\ & \text { and social networking; in Schaie KW, Willis } \\ & \text { SL (eds): Handbook of the Psychology of Ag- } \\ & \text { ing, ed 8. Amsterdam, Elsevier, 2016, pp 389- } \\ & 407 . \\ 3 \text { Charness N, Boot WR: Aging and informa- } & \text { tion technology use: potential and barriers. } \\ & \text { Curr Dir Psychol Sci 2009;18:253-258. }\end{aligned}$

Gerontology 2017;63:385-392

DOI: $10.1159 / 000471802$ 
4 Fisk AD, Rogers WA, Charness N, Czaja SJ, Sharit J: Designing for Older Adults: Principles and Creative Human Factors Approaches, ed 2. Human Factors \& Aging Series. Boca Raton, CRC Press, 2009.

5 Lindenberger U, Lövdén M, Schellenbach M, Li SC, Krüger A: Psychological principles of successful aging technologies: a mini-review. Gerontology 2008;54:59-68.

6 Elliot AJ, Mooney CJ, Douthit KZ, Lynch MF: Predictors of older adults' technology use and its relationship to depressive symptoms and well-being. J Gerontol B Psychol Sci Soc Sci 2014;69:667-677.

7 Gell NM, Rosenberg DE, Demiris G, LaCroix AZ, Patel KV: Patterns of technology use among older adults with and without disabilities. Gerontologist 2015;55:412-421.

8 Kamin ST, Lang FR: Cognitive functions buffer age differences in technology ownership. Gerontology 2016;62:238-246.

9 Melenhorst AS, Rogers WA, Bouwhuis DG: Older adults' motivated choice for technological innovation: evidence for benefit-driven selectivity. Psychol Aging 2006;21:190-195.

10 Melenhorst AS, Bouwhuis DG: When do older adults consider the internet? An exploratory study of benefit perception. Gerontechnology 2004;3:89-101.

11 Chen K, Chan AHS: A review of technology acceptance by older adults. Gerontechnology 2011;10:1-12.

12 Merkel S, Enste P, Hilbert J, Chen K, Chan AHS, Kwon S: Technology acceptance and aging; in Kwon S (ed): Gerontechnology: Research, Practice, and Principles in the Field of Technology and Aging. New York, Springer, 2016, pp 335-349.

13 Lawton MP: Behavior-relevant ecological factors; in Schaie KW, Schooler C (eds): Social Structure And Aging: Psychological Processes. Hillsdale, Erlbaum, 1989, pp 57-78.

14 Kamin ST, Lang FR: The Subjective Technology Adaptivity Inventory (STAI): a motivational measure of technology usage in old age. Gerontechnology 2013;12:16-25.
15 Heckhausen J, Wrosch C, Schulz R: A motivational theory of life-span development Psychol Rev 2010;117:32-60.

16 Camp LJ, Lorenzen-Huber L: Privacy implications of aware, active, and adaptive technologies; in Kwon S (ed): Gerontechnology: Research, Practice, and Principles in the Field of Technology and Aging. New York, Springer, 2016, pp 91-114.

17 Boise L, Wild K, Mattek N, Ruhl M, Dodge $\mathrm{HH}$, Kaye J: Willingness of older adults to share data and privacy concerns after exposure to unobtrusive in-home monitoring. Gerontechnology 2013;11:428-435.

18 Czaja SJ, Charness N, Fisk AD, Hertzog C, Nair SN, Rogers WA, Sharit J: Factors predicting the use of technology: findings from the Center for Research and Education on Aging and Technology Enhancement (CREATE). Psychol Aging 2006;21:333-352.

19 Laguna K, Babcock RL: Computer anxiety in young and older adults: implications for human-computer interactions in older populations. Comput Human Behav 1997;13:317326.

20 McElroy JC, Hendrickson AR, Townsend AM, DeMarie SM: Dispositional factors in Internet use: personality versus cognitive style. MIS Q 2007;31:809-820.

21 Correa T, Hinsley AW, de Zúñiga HG: Who interacts on the Web?: The intersection of users' personality and social media use. Comput Human Behav 2010;26:247-253.

22 Barnett T, Pearson AW, Pearson R, Kellermanns FW: Five-factor model personality traits as predictors of perceived and actual usage of technology. Eur J Inf Syst 2015;24:374390.

23 McAdams DP, Pals JL: A new Big Five: fundamental principles for an integrative science of personality. Am Psychol 2006;61:204-217.
24 Iwarsson S, Slaug B: Housing Enabler - A Method for Rating/Screening and Analysing Accessibility Problems in Housing, ed 2. Lund, Veten \& Skapen HB \& Slaug Enabling Development, 2010.

25 Lang FR, John D, Lüdtke O, Schupp J, Wagner GG: Short assessment of the Big Five: robust across survey methods except telephone interviewing. Behav Res Methods 2011;43:548567.

26 Alderman H, Behrman JR, Kohler HP, Maluccio JA, Watkins SC: Attrition in longitudinal household survey data: some tests for three developing-country samples. Demogr Res 2001;5:79-124.

27 StataCorp: Stata Statistical Software: Release 14. College Station, StataCorp LP, 2015.

28 StataCorp: Stata Multiple-Imputation Reference Manual: Release 14. College Station, Stata Press, 2015.

29 Lang FR, Rohr MK, Williger B: Modeling success in life-span psychology: the principles of selection, optimization, and compensation; in Fingerman KL, Berg CA, Smith J, Antonucci TC (eds): Handbook of Life-Span Development. New York, Springer Publishing Company, 2011, pp 57-85.

30 Wahl HW, Iwarsson S, Oswald F: Aging well and the environment: toward an integrative model and research agenda for the future. Gerontologist 2012;52:306-316.

31 Wahl HW, Oswald F: Theories of environmental gerontology: old and new avenues for person-environmental views of aging; in Bengtson VL, Settersten RA (eds): Handbook of Theories of Aging, ed 3. New York, Springer, 2016, pp 621-641.

32 Kamin ST, Luft T, Miehling J, Williger B, Lang FR, Wartzack S: Subjektive Adaptionsfähigkeit im Kontext der alternssensiblen Produktentwicklung; in Krause D, Paetzold K, Wartzack S (eds): Design for X. Beiträge zum 25. DfX Symposium 2014. Hamburg, TuTech Verlag, 2014, pp 99-110. 